East Asian Math. J.

Vol. 30 (2014), No.3, pp. 249-258

http://dx.doi.org/10.7858/eamj.2014.015

\title{
ENERGY DECAY RATES FOR THE KIRCHHOFF TYPE WAVE EQUATION WITH BALAKRISHNAN-TAYLOR DAMPING AND ACOUSTIC BOUNDARY
}

\author{
YONG HAN KANG
}

\begin{abstract}
In this paper, we study uniform exponential stabilization of the vibrations of the Kirchhoff type wave equation with BalakrishnanTaylor damping and acoustic boundary in a bounded domain in $R^{n}$. To stabilize the systems, we incorporate separately, the passive viscous damping in the model as like Kang[14]. Energy decay rates are obtained by the uniform exponential stability of solutions by using multiplier technique.
\end{abstract}

\section{Introduction}

In this paper, we consider the uniform stability of mathematical problems governed by the following a nonlinear wave equations of the Kirchhoff type wave equation with Balakrishnan-Taylor damping and acoustic boundary conditions:

$$
\begin{gathered}
\left|u^{\prime}\right|^{\rho} u^{\prime \prime}+2 \delta u^{\prime}=\left(a^{2}+b \int_{\Omega}|\nabla u|^{2} d x+\sigma \int_{\Omega} \nabla u \cdot \nabla u^{\prime} d x\right) \triangle u \text { in } \Omega \times R^{+}, \\
u=0 \text { on } \Gamma_{0} \times R^{+}, \\
\left(a^{2}+b \int_{\Omega}|\nabla u|^{2} d x+\sigma \int_{\Omega} \nabla u \cdot \nabla u^{\prime} d x\right) \frac{\partial u}{\partial \nu}=y^{\prime} \text { on } \Gamma_{1} \times R^{+}, \\
u^{\prime}+p(x) y^{\prime}+q(x) y=0 \text { on } \Gamma_{1} \times R^{+}, \\
u(0)=u_{0}, u^{\prime}(0)=u_{1} \text { in } \Omega
\end{gathered}
$$

where $\Omega$ is a bounded, connected set in $R^{n}(n \geq 1)$ having a smooth boundary $\Gamma=\partial \Omega$, consisting of two parts $\Gamma_{0}$ and $\Gamma_{1}$ such that $\overline{\Gamma_{0}} \cup \overline{\Gamma_{1}}=\Gamma$. Primes denote the time derivaites, $\Delta$ the laplacian in $R^{n}$ taken in space variables, $\nu$ the unit normal of $\Gamma$ pointing towards exterior of $\Omega$ and $R^{+}:=(0, \infty)$. The parameters $\delta>0$ is a small passive viscous damping coefficient, and $a>0, b>$ $0, \sigma>0, \rho>0$ are constant real numbers. $p$ and $q$ are functions satisfying some conditions to be specified later. Physically, the integro-differential equations

Received January 16, 2014; Accepted January 22, 2014.

2010 Mathematics Subject Classification. 35L70; 35B40, 76Exx.

Key words and phrases. Kirchhoff type, Energy decay, Balakrishnan-Taylor damping, Acoustic boundary, Stabilization, Lyapunov functional.

This work was financially supported by the research foundation of the Catholic University of Daegu in 2012 year; 20121072. 
(1)-(5) occurs in the study of vibrations of damped flexible space structures in bounded domain in $R^{n}$. The nonlinear term $\left|u^{\prime}\right|^{\rho} u^{\prime \prime}, \rho>0$ is modeled materials whose density depends on the velocity $u^{\prime}$. The term $2 \delta u^{\prime}$ is the distributed damping of passive viscous type. The boundary conditions considered here are of mixed Dirichlet and Neumann type and acoustic boundary. And also The model in hand, with Balakrishnan-Taylor damping $(\sigma>0)$ and $\rho=1$, was initially proposed by Balakrishnan and Taylor in 1989 [16] and Bass and Zes [17]. On the other hand, for the conditions $\rho=1, \sigma=0$, Kang was worked in 2012 [14]. The analytical studies in the area of stabilization of distributed parameter system is currently of interest in view of application to vibration control of various structural elements. The phenomenon was first observed by Hunton as reported by Harrison [7]. The nonlinear model like (1) for transverse vibrations was originally derived by Kirchhoff [3]. Beale and Rosencrans[5] introduced acoustic boundary conditions of the general form

$$
\begin{aligned}
& \frac{\partial u}{\partial \nu}=y^{\prime} \text { on } \Gamma_{1} \times R^{+} \\
& \gamma u^{\prime}+m(x) y^{\prime \prime}+p(x) y^{\prime}+q(x) y=0 \text { on } \Gamma_{1} \times R^{+} .
\end{aligned}
$$

Recently, wave equations with acoustic boundary conditions have been treated by many authors [4],[5],[6],[8],[9],[10],[11],[12],[13]. In [4], the authors studied the nonlinear wave equations

$$
\begin{aligned}
& u^{\prime \prime}-M\left(\int_{\Omega}|u|^{2} d x\right) \Delta u+\left|u^{\prime}\right|^{\alpha} u^{\prime}=0 \text { in } \Omega \times R^{+}, \\
& u=0 \text { on } \Gamma_{0} \times R^{+} \\
& \frac{\partial u}{\partial \nu}=y^{\prime} \text { on } \Gamma_{1} \times R^{+}, \\
& \gamma u^{\prime}+m(x) y^{\prime \prime}+p(x) y^{\prime}+q(x) y=0 \text { on } \Gamma_{1} \times R^{+} .
\end{aligned}
$$

They proved the existence of solutions, but gave no decay rate for solutions. As regards uniform decay rates for solutions to problems with acoustic boundary conditions, there are not much literature [2],[4],[9],[10],[11]. Frota and Larkin[9] established global solvability and decay estimates for a linear wave equation with boundary conditions

$$
\begin{aligned}
& \frac{\partial u}{\partial \nu}=h(x) y^{\prime} \text { on } \Gamma_{1} \times R^{+} \\
& \gamma u^{\prime}+p(x) y^{\prime}+q(x) y=0 \text { on } \Gamma_{1} \times R^{+} .
\end{aligned}
$$

In this paper we are motivated by boundary conditions of Park[4] and results of Gorain[1], Kang[14] and Zarai and Tatar[15]. The aim of this paper is to study uniform stabilization of the generalized nonlinear Kirchhoff type wave equations governed by (1)-(5) with the mixed boundary conditions. To our knowledge, this problem has not been considered by predecessors and is studied first, as a Kirchhoff model, in this paper. The plan of this paper as follows. In section 2, we give some notation, some conditions and material needed for our work. In 
section 3, we drive the uniform stability on account of viscous damping with acoustic boundary. The notation used in this paper is standard and can be found in Gorain[1].

\section{Preliminaries and some notations}

In this section, we present some notations and some material in the proof of our result. Throughout this paper, we use the notation $V=\left\{u \in H^{1}(\Omega)\right.$ : $u=0$ on $\left.\Gamma_{0}\right\}$ the subspace of the classical Sobolev space $H^{1}(\Omega)$ of real valued functions of order one. Let $k$ be the smallest positive constant independent of $t$ (depends only on $\Omega$ ) satisfying the Poincare inequality

$$
\int_{\Omega} u^{2} d x \leq k \int_{\Omega}|\nabla u|^{2} d x \text { for every } u \in V .
$$

And also let $\bar{k}$ be the smallest positive constant independent of $t$ (depends only on $\Gamma_{1}$ ) satisfying the embeding inequality

$$
\int_{\Gamma_{1}} u^{2} d x \leq \bar{k} \int_{\Omega}|\nabla u|^{2} d x \text { for every } u \in V .
$$

We assume that

$$
\rho \text { satisfies } 0<\rho \leq \frac{n}{n-2} \text {, if } n \geq 3 \text { or } \rho>0 \text {, if } n=1,2 \text {. }
$$

and since $V \hookrightarrow L^{\rho+2}(\Omega)$,

$$
\text { there exist a positive constant } K \text { such that }\|u\|_{\rho+2} \leq K\|\nabla u\|_{2} \text {. }
$$

For the functions $p$ and $q$, we assume that $p, q \in C\left(\Gamma_{1}\right)$ and $p(x)>0$ and $q(x)>0$ for all $x \in \Gamma_{1}$. This assumption implies that there exist positive constants $p_{i}, q_{i}(i=0,1)$ such that

$$
p_{0} \leq p(x) \leq p_{1}, q_{0} \leq q(x) \leq q_{1} \text { for all } x \in \Gamma_{1} .
$$

By using Gälerkin's approximation, we can obtain the following existence result for the solution subject to (1)-(5) under the conditions on $p$ and $q$ as above. For the initial data $\left(u_{0}, u_{1}\right) \in\left(V \cap H^{2}(\Omega)\right) \times V$, there exists a unique pair of functions $(u, y)$, which is a solution to the problem (1)-(5) in the class

$$
\begin{array}{cl}
u \in L^{\infty}\left(0, T ; V \times H^{2}(\Omega)\right), & u^{\prime} \in L^{\infty}(0, T ; V), \\
u^{\prime \prime} \in L^{\infty}\left(0, T ; L^{2}(\Omega)\right), & y, y^{\prime} \in L^{2}\left(0, \infty ; L^{2}\left(\Gamma_{1}\right)\right) .
\end{array}
$$

In the order to state our main results, we define the energy of problem (1)-(5) by

$$
\begin{aligned}
E(t)= & \frac{1}{\rho+2} \int_{\Omega}\left|u^{\prime}\right|^{\rho+2} d x+\frac{a^{2}}{2} \int_{\Omega}|\nabla u|^{2} d x \\
& +\frac{b}{4}\left(\int_{\Omega}|\nabla u|^{2} d x\right)^{2}+\frac{1}{2} \int_{\Gamma_{1}} q(x)(y)^{2} d \Gamma .
\end{aligned}
$$




\section{Stability on account of passive viscous damping}

If we differentiate (11) with respect to $t$ and use the governing Eq.(1) we obtain

$$
\begin{aligned}
E^{\prime}(t)= & \int_{\Omega}\left|u^{\prime}\right|^{\rho} u^{\prime \prime} u^{\prime} d x+a^{2} \int_{\Omega} \nabla u \cdot \nabla u^{\prime} d x \\
+ & b\left(\int_{\Omega}|\nabla u|^{2} d x\right) \int_{\Omega} \nabla u \cdot \nabla u^{\prime} d x+\int_{\Gamma_{1}} q(x) y y^{\prime} d \Gamma \\
= & \int_{\Omega}\left\{-2 \delta u^{\prime}+\left(a^{2}+b \int_{\Omega}|\nabla u|^{2} d x+\sigma \int_{\Omega} \nabla u \cdot \nabla u^{\prime} d x\right) \triangle u\right\} u^{\prime} d x \\
& +a^{2} \int_{\Omega} \nabla u \cdot \nabla u^{\prime} d x+b\left(\int_{\Omega}|\nabla u|^{2} d x\right) \int_{\Omega} \nabla u \cdot \nabla u^{\prime} d x+\int_{\Gamma_{1}} q(x) y y^{\prime} d \Gamma .
\end{aligned}
$$

Application of Green's formula, using the boundary conditions (2)-(5) and then a simplification, we get

$$
\begin{aligned}
E^{\prime}(t)= & -2 \delta \int_{\Omega}\left|u^{\prime}\right|^{2} d x-\sigma\left(\int_{\Omega} \nabla u \cdot \nabla u^{\prime} d x\right)^{2}+\int_{\Gamma_{1}}\left(u^{\prime}+q(x) y\right) y^{\prime} d \Gamma \\
=- & 2 \delta \int_{\Omega}\left|u^{\prime}\right|^{2} d x-\sigma\left(\int_{\Omega} \nabla u \cdot \nabla u^{\prime} d x\right)^{2} \\
& -\int_{\Gamma_{1}} p(x)\left(y^{\prime}\right)^{2} d \Gamma<0 \quad \forall t \in R^{+}
\end{aligned}
$$

We see from (12) that the energy $E$ is a decreasing function of time and hence

$$
E(t) \leq E(0) \quad \forall t \geq 0
$$

where

$$
\begin{aligned}
E(0)= & \frac{1}{\rho+2} \int_{\Omega}\left|u_{1}\right|^{\rho+2} d x+\frac{a^{2}}{2} \int_{\Omega}\left|\nabla u_{0}\right|^{2} d x+\frac{b}{4}\left(\int_{\Omega}\left|\nabla u_{0}\right|^{2} d x\right)^{2} \\
& +\frac{1}{2} \int_{\Gamma_{1}} q(x)(y(x, 0))^{2} d \Gamma .
\end{aligned}
$$

Under what conditions does this energy $E$ decay with time uniformly? An affirmative answer is contained in the following theorem.

Theorem 3.1. If $u=u(x, t)$ is a regular solution of the system (1)-(5) with initial values $\left(u_{0}, u_{1}\right) \in V \times L^{2}(\Omega)$, then the energy $E(t)$ of the system defined by (11) satisfies

$$
E(t)<M e^{-\mu t} E(0), t \in(0, \infty)
$$

for some real constants $M>1(40)$ and $\mu>0(37)$ and depend on $\epsilon(35)$.

Firstly, we prove the following lemma. 
Lemma 3.2. For every solution $u=u(x, t)$ of the system (1)-(5), the time derivative of the functional $G$ defined by

$$
G(t)=\frac{1}{\rho+1} \int_{\Omega}\left|u^{\prime}\right|^{\rho} u^{\prime} u d x+\int_{\Omega} \delta u^{2} d x+\frac{\sigma}{4} \int_{\Gamma_{1}} u y d \Gamma+\frac{1}{2} \int_{\Gamma_{1}} p(x) y^{2} d \Gamma
$$

satisfies

$$
\begin{aligned}
G^{\prime}(t) \leq & \frac{1}{\rho+1} \int_{\Omega}\left|u^{\prime}\right|^{\rho+2} d x+2 \int_{\Gamma_{1}} u y^{\prime} d \Gamma-\int_{\Gamma_{1}} q(x)(y)^{2} d \Gamma \\
& -a^{2} \int_{\Omega}|\nabla u|^{2} d x-b\left(\int_{\Omega}|\nabla u|^{2} d x\right)^{2}+\left.\frac{\sigma}{4}\left(\int_{\Omega} \nabla u \cdot u^{\prime} d x\right)\right|^{2}, t \in R^{+}
\end{aligned}
$$

Proof. If we differentiate (14) with respect to $t$ and replace $u^{\prime \prime}$ by the relation (1), then we get

$$
\begin{aligned}
G^{\prime}(t) & =\int_{\Omega}\left|u^{\prime}\right|^{\rho} u^{\prime \prime} u d x+\frac{1}{\rho+1} \int_{\Omega}\left|u^{\prime}\right|^{\rho+2} d x+2 \delta \int_{\Omega} u^{\prime} u d x \\
+ & \sigma\left(\int_{\Omega}|\nabla u|^{2} d x\right) \int_{\Omega} \nabla u \cdot \nabla u^{\prime} d x+\int_{\Gamma_{1}}\left(u^{\prime} y+u y^{\prime}\right) d \Gamma+\int_{\Gamma_{1}} p(x) y y^{\prime} d \Gamma \\
= & \int_{\Omega}\left(\left|u^{\prime}\right|^{\rho} u^{\prime \prime}+2 \delta u^{\prime}\right) u d x+\frac{1}{\rho+1} \int_{\Omega}\left|u^{\prime}\right|^{\rho+2} d x \\
& +\sigma\left(\int_{\Omega}|\nabla u|^{2} d x\right) \int_{\Omega} \nabla u \cdot \nabla u^{\prime} d x+\int_{\Gamma_{1}}\left(u^{\prime} y+u y^{\prime}\right) d \Gamma+\int_{\Gamma_{1}} p(x) y y^{\prime} d \Gamma \\
= & \int_{\Omega}\left\{\left(a^{2}+b \int_{\Omega}|\nabla u|^{2} d x+\sigma \int_{\Omega}|\nabla u|^{2} d x\right) \triangle u\right\} u d x+\frac{1}{\rho+1} \int_{\Omega}\left|u^{\prime}\right|^{\rho+2} d x \\
& +\sigma\left(\int_{\Omega}|\nabla u|^{2} d x\right) \int_{\Omega} \nabla u \cdot \nabla u^{\prime} d x+\int_{\Gamma_{1}} u y^{\prime} d \Gamma+\int_{\Gamma_{1}} y\left(u^{\prime}+p(x) y^{\prime}\right) d \Gamma
\end{aligned}
$$

Applying Green's formula and boundary conditions, we have

$$
\begin{aligned}
G^{\prime}(t)= & \int_{\Gamma_{1}}\left(a^{2}+b \int_{\Omega}|\nabla u|^{2} d x+\sigma \int_{\Omega}|\nabla u|^{2} d x\right) \frac{\partial u}{\partial \nu} u d \Gamma \\
& -\int_{\Omega}\left(a^{2}+b \int_{\Omega}|\nabla u|^{2} d x+\sigma \int_{\Omega}|\nabla u|^{2} d x\right) \nabla u \cdot \nabla u d x \\
& +\frac{1}{\rho+1} \int_{\Omega}\left|u^{\prime}\right|^{\rho+2} d x+\sigma\left(\int_{\Omega}|\nabla u|^{2} d x\right) \int_{\Omega} \nabla u \cdot \nabla u^{\prime} d x \\
& +\int_{\Gamma_{1}} u y^{\prime} d \Gamma+\int_{\Gamma_{1}} y\left(u^{\prime}+p(x) y^{\prime}\right) d \Gamma .
\end{aligned}
$$


Using the boundary condition(5) and Young's inequality, relation (17) can be written as

$$
\begin{aligned}
& G^{\prime}(t)=\frac{1}{\rho+1} \int_{\Omega}\left|u^{\prime}\right|^{\rho+2} d x-a^{2} \int_{\Omega}|\nabla u|^{2} d x-b\left(\int_{\Omega}|\nabla u|^{2} d x\right)^{2} \\
&-\sigma\left(\int_{\Omega}|\nabla u|^{2} d x\right)^{2}+\sigma\left(\int_{\Omega}|\nabla u|^{2} d x\right)\left(\int_{\Omega} \nabla u \cdot \nabla u^{\prime} d x\right) \\
&+2 \int_{\Gamma_{1}} u y^{\prime} d \Gamma+\int_{\Gamma_{1}} y\left(u^{\prime}+p(x) y^{\prime}\right) d \Gamma \\
& \leq \frac{1}{\rho+1} \int_{\Omega}\left|u^{\prime}\right|^{\rho+2} d x+2 \int_{\Gamma_{1}} u y^{\prime} d \Gamma-\int_{\Gamma_{1}} q(x)(y)^{2} d \Gamma \\
&-a^{2} \int_{\Omega}|\nabla u|^{2} d x-b\left(\int_{\Omega}|\nabla u|^{2} d x\right)^{2}+\frac{\sigma}{4}\left(\int_{\Omega} \nabla u \cdot \nabla u^{\prime} d x\right)^{2}, \quad \forall t \in R^{+} .
\end{aligned}
$$

Hence the proof of lemma complete.

Proof of Theorem 1. We introduce an energy like Lyapunov functional $V$ by

$$
V(t)=E(t)+\epsilon G(t) \text { for } t \geq 0 .
$$

Now, using the Cauchy-Schwarz's inequality, the Hölder inequality, the Poincare inequality (6)-(9) and the defined of energy (11), we obtain estimate as follow

$$
\begin{aligned}
& \left.\left.\left|\frac{1}{\rho+1} \int_{\Omega}\right| u^{\prime}\right|^{\rho} u^{\prime} u d x\left|\leq \frac{1}{\rho+1} \int_{\Omega}\right| u^{\prime}\right|^{\rho+1}|u| d x \\
& \leq \frac{1}{\rho+1}\left(\int_{\Omega}\left|u^{\prime}\right|^{\rho+2} d x\right)^{\frac{\rho+1}{\rho+2}}\left(\int_{\Omega}|u|^{\rho+2} d x\right)^{\frac{1}{\rho+2}} \\
& \leq \frac{1}{\rho+2} \int_{\Omega}\left|u^{\prime}\right|^{\rho+2} d x+\frac{1}{(\rho+1)(\rho+2)} \int_{\Omega}|u|^{\rho+2} d x \\
& \leq \frac{1}{\rho+2}\left\|u^{\prime}\right\|_{\rho+2}^{\rho+2}+\frac{\tilde{k}^{\rho+2}}{(\rho+1)(\rho+2)}\|\nabla u\|^{\rho+2} \\
& \leq \frac{1}{\rho+2}\left\|u^{\prime}\right\|_{\rho+2}^{\rho+2}+\frac{\tilde{k}^{\rho+2}}{(\rho+1)(\rho+2)}\left(\frac{2 E(0)}{a^{2}}\right)^{\frac{\rho}{2}}\|\nabla u\|^{2} \\
& \leq\left\{1+\frac{\tilde{k}^{\rho+2}}{(\rho+1)(\rho+2)}\left(\frac{2 E(0)}{a^{2}}\right)^{\frac{\rho}{2}} \frac{2}{a^{2}}\right\} E(t) \\
& 0 \leq \delta \int_{\Omega} u^{2} d x \leq \delta k \int_{\Omega}|\nabla u|^{2} d x=\frac{2 \delta k}{a^{2}} \frac{a^{2}}{2} \int_{\Omega}|\nabla u|^{2} d x \leq \frac{2 \delta k}{a^{2}} E(t), \\
& \left|\int_{\Gamma_{1}} u y d \Gamma\right| \leq \int_{\Gamma_{1}} \frac{1}{2 q(x)} u^{2} d \Gamma+\frac{1}{2} \int_{\Gamma_{1}} q(x) y^{2} d \Gamma \\
& \leq \frac{\bar{k}}{2 q_{0}} \int_{\Omega}|\nabla u|^{2} d x+\frac{1}{2} \int_{\Gamma_{1}} q(x) y^{2} d \Gamma \leq\left(\frac{\bar{k}}{a^{2} q_{0}}+1\right) E(t),
\end{aligned}
$$




$$
\frac{1}{2} \int_{\Gamma_{1}} p(x) y^{2} d \Gamma \leq \frac{p_{1}}{2 q_{0}} \int_{\Gamma_{1}} q(x) y^{2} d \Gamma \leq \frac{p_{1}}{q_{0}} E(t),
$$

and

$$
\frac{\sigma}{4}\left(\int_{\Omega}|\nabla u|^{2} d x\right)^{2} d x \leq \frac{\sigma}{b} E(t) .
$$

Thus the inequality (20)-(24) and (14) yield for $G$ that estimates

$$
\begin{aligned}
& -\left[2+\frac{2 K^{\rho+2}}{a^{2}(\rho+1)(\rho+2)}\left(\frac{2 E(0)}{a^{2}}\right)^{\rho / 2}+\frac{\bar{k}}{a^{2} q_{0}}\right] E(t) \leq G(t) \\
\leq & {\left[2+\frac{2 K^{\rho+2}}{a^{2}(\rho+1)(\rho+2)}\left(\frac{2 E(0)}{a^{2}}\right)^{\rho / 2}+\frac{2 \delta k}{a^{2}}+\frac{\bar{k}}{a^{2} q_{0}}+\frac{p_{1}}{q_{0}}+\frac{\sigma}{b}\right] E(t) . }
\end{aligned}
$$

Then it follows from (25) that

$$
\begin{gathered}
\left\{1-\epsilon\left(2+\frac{K^{\rho+2}}{(\rho+1)(\rho+2)}\left(\frac{2 E(0)}{a^{2}}\right)^{\frac{\rho}{2}} \frac{2}{a^{2}}+\frac{\bar{k}}{a^{2} q_{0}}\right)\right\} E(t) \leq V(t) \leq \\
\left\{1+\epsilon\left(2+\frac{K^{\rho+2}}{(\rho+1)(\rho+2)}\left(\frac{2 E(0)}{a^{2}}\right)^{\frac{\rho}{2}} \frac{2}{a^{2}}+\frac{2 \delta k}{a^{2}}+\frac{\bar{k}}{a^{2} q_{0}}+\frac{p_{1}}{q_{0}}+\frac{\sigma}{b}\right)\right\} E(t) \quad \forall t \geq 0,
\end{gathered}
$$

where we assume that

$$
0<\epsilon<\frac{1}{2+\frac{K \rho+2}{(\rho+1)(\rho+2)}\left(\frac{2 E(0)}{a^{2}}\right)^{\frac{\rho}{2}} \frac{2}{a^{2}}+\frac{\bar{k}}{a^{2} q_{0}}},
$$

so that left hand side of (26) is positive. Let

$$
M_{1}:=2+\frac{K^{\rho+2}}{(\rho+1)(\rho+2)}\left(\frac{2 E(0)}{a^{2}}\right)^{\frac{\rho}{2}} \frac{2}{a^{2}}+\frac{\bar{k}}{a^{2} q_{0}}
$$

and

$$
M_{2}:=2+\frac{K^{\rho+2}}{(\rho+1)(\rho+2)}\left(\frac{2 E(0)}{a^{2}}\right)^{\frac{\rho}{2}} \frac{2}{a^{2}}+\frac{2 \delta k}{a^{2}}+\frac{\bar{k}}{a^{2} q_{0}}+\frac{p_{1}}{q_{0}}+\frac{\sigma}{b} .
$$

Next, differentiating $V(t)$ (defined by (19)) with respect to $t$ using expression $E^{\prime}(t)$ (defined by (12)) and Lemma 3.1, we have

$$
\begin{aligned}
& V^{\prime}(t) \leq-2 \delta \int_{\Omega}\left|u^{\prime}\right|^{2} d x-\sigma\left(\int_{\Omega} \nabla u \cdot \nabla u^{\prime} d x\right)^{2}-\int_{\Gamma_{1}} p(x)\left(y^{\prime}\right)^{2} d \Gamma \\
&+\epsilon\left\{\frac{1}{\rho+1} \int_{\Omega}\left|u^{\prime}\right|^{\rho+2} d x+2 \int_{\Gamma_{1}} u y^{\prime} d \Gamma-\int_{\Gamma_{1}} q(x)(y)^{2} d \Gamma\right. \\
&\left.\quad-a^{2} \int_{\Omega}|\nabla u|^{2} d x-b\left(\int_{\Omega}|\nabla u|^{2} d x\right)^{2}+\frac{\sigma}{4}\left(\int_{\Omega} \nabla u \cdot \nabla u^{\prime} d x\right)^{2}\right\}
\end{aligned}
$$


Now, using the Cauchy-Schwarz's inequality, the Poincare inequality, the conditions (7)-(8) and the definition of energy (9), we obtain estimate

$$
\begin{gathered}
\left|2 \epsilon \int_{\Gamma_{1}} u y^{\prime} d x\right| \leq \int_{\Gamma_{1}} p(x)\left(y^{\prime}\right)^{2} d \Gamma+\epsilon^{2} \int_{\Gamma_{1}} \frac{1}{p(x)} u^{2} d \Gamma \\
\leq \int_{\Gamma_{1}} p(x)\left(y^{\prime}\right)^{2} d \Gamma+\frac{2 \bar{k} \epsilon^{2}}{a^{2} p_{0}} \frac{a^{2}}{2} \int_{\Omega}|\nabla u|^{2} d x \\
\leq \int_{\Gamma_{1}} p(x)\left(y^{\prime}\right)^{2} d \Gamma+\frac{2 \bar{k} \epsilon^{2}}{a^{2} p_{0}} E(t) .
\end{gathered}
$$

From (28)-(29), we have

$$
\begin{aligned}
V^{\prime}(t) & \leq-2 \delta \int_{\Omega}\left|u^{\prime}\right|^{2} d x-\sigma\left(1-\frac{\epsilon}{4}\right)\left(\int_{\Omega} \nabla u \cdot \nabla u^{\prime} d x\right)^{2} \\
& +\frac{\epsilon}{\rho+1} \int_{\Omega}\left|u^{\prime}\right|^{\rho+2} d x+\frac{2 \bar{k} \epsilon^{2}}{a^{2} p_{0}} E(t)-\epsilon-\int_{\Gamma_{1}} p(x)\left(y^{\prime}\right)^{2} d \Gamma \\
& -a^{2} \epsilon \int_{\Omega}|\nabla u|^{2} d x-b \epsilon\left(\int_{\Omega}|\nabla u|^{2} d x\right)^{2} \\
= & -2 \delta \int_{\Omega}\left|u^{\prime}\right|^{2} d x+\frac{\epsilon}{\rho+1} \int_{\Omega}\left|u^{\prime}\right|^{\rho+2} d x-\sigma\left(1-\frac{\epsilon}{4}\right)\left(\int_{\Omega} \nabla u \cdot \nabla u^{\prime} d x\right)^{2} \\
& -2 \epsilon\left(1-\frac{\bar{k} \epsilon}{a^{2} p_{0}}\right) E(t)-\frac{b \epsilon}{2}\left(\int_{\Omega}|\nabla u|^{2} d x\right)^{2} .
\end{aligned}
$$

Since $L^{\rho+2}(\Omega) \hookleftarrow L^{2}(\Omega)$ and by the definition of energy (11), we note that

$$
\begin{aligned}
& \left\|u^{\prime}\right\|_{2} \leq\left\|u^{\prime}\right\|_{\rho+2}, \\
& \left\|u^{\prime}\right\|_{\rho+2}^{\rho} \leq[(\rho+2) E(0)]^{\frac{\rho}{\rho+2}} .
\end{aligned}
$$

And also we can take sufficiently small $\epsilon$ satisfy

$$
0<\epsilon<4, \quad 0<\epsilon<\frac{a^{2} p_{0}}{k}, \quad 0<\epsilon<\frac{2 \delta(\rho+1)(\rho+2)}{3 \rho+4}\{(\rho+2) E(0)\}^{-\frac{\rho}{\rho+2}},
$$

since

$$
\left\|u^{\prime}\right\|_{\rho+2}^{\rho} \leq\{(\rho+2) E(0)\}^{\rho /(\rho+2)}, \quad-2 \delta\left\|u^{\prime}\right\|^{2}+\epsilon \frac{3 \rho+4}{(\rho+1)(\rho+2)}\left\|u^{\prime}\right\|_{\rho+2}^{\rho+2} .
$$

From (31)-(33) and (30), we obtain

$$
\begin{aligned}
V^{\prime}(t) & \leq-2 \epsilon\left(1-\frac{\bar{k} \epsilon}{a^{2} p_{0}}\right) E(t) \\
& -\sigma\left(1-\frac{\epsilon}{4}\right)\left(\int_{\Omega} \nabla u \cdot \nabla u^{\prime} d x\right)^{2}-\frac{b \epsilon}{2}\left(\int_{\Omega}|\nabla u|^{2} d x\right)^{2} \\
& -\left(2 \delta \int_{\Omega}\left|u^{\prime}\right|^{2} d x-\frac{\epsilon(3 \rho+4)}{(\rho+1)(\rho+2)} \int_{\Omega}\left|u^{\prime}\right|^{\rho+2} d x\right) \\
& <-2 \epsilon\left(1-\frac{\bar{k} \epsilon}{a^{2} p_{0}}\right) E(t), \quad \forall t>0
\end{aligned}
$$


where we assume that

$$
0<\epsilon<\min \left\{4, M_{1}^{-1}, \frac{a^{2} p_{0}}{\bar{k}}, \frac{2 \delta(\rho+1)(\rho+2)}{3 \rho+4}\{(\rho+2) E(0)\}^{-\frac{\rho}{\rho+2}}\right\} .
$$

With the help of (26), the above yields the differential inequality

$$
V^{\prime}(t)+\mu V(t)<0 \quad \forall t \in R^{+},
$$

where

$$
0<\mu=\frac{2 \epsilon\left(a^{2} p_{0}-\bar{k} \epsilon\right)}{a^{2} p_{0}\left(1+\epsilon M_{2}\right)} .
$$

Multiplying (36) by $e^{\mu t}$ and integrating over the time interval $[0, t]$, we get the estimate

$$
V(t)<e^{-\mu t} V(0) \quad \forall t \in R^{+} .
$$

Invoking the inequality (26) again in (38), we have

$$
E(t)<M e^{-\mu t} E(0) \quad \forall t \in R^{+},
$$

where

$$
M=\frac{1+\epsilon M_{2}}{1-\epsilon M_{1}}>1 .
$$

The finishes the proof of the theorem.

\section{References}

[1] G.C. Gorain, Exponential eneragy decay estimates for the solutions of $n$-dimensional Kirchhoff type wave equation, Applied Mathematics and Computation 117 (2006), 235242.

[2] M.A. Horn, Exact controllability and uniform stabilization of the Kirchhoff plate equation with boundary feedback acting via bending moments, J. Math. Anal. Appl. 167 (1992), 557-581.

[3] G. Kirchhoff, Vorlesungen übear Mathematische Physik, Mechanik(Teubner) 1977.

[4] J.Y. Park and S.H. Park, Decay rate estimates for wave equations of memory type with acoustic boundary conditions, Nonlinear Analysis : Theory, methods and Applications 74 (2011), no. 3, 993-998

[5] J.T. Beal and S.I. Rosencrans, Acoustic boundary conditions, Bull. Amer. Math. Soc. 80 (1974), 1276-1278.

[6] C.L. Frota and J.A. Goldstein, Some nonlinear wave equations with acoustic boundary conditions, J. Differ. Equ. 164 (2000), 92-109.

[7] H. Harrison, Plane and circular motion of a string, J. Acoust. Soc. Am. 20 (1948), 874-875.

[8] A.T. Cousin, C.L. Frota and N.A. Larkin, On a system of Klein-Gordon type equations with acoustic boundary conditions, J. Math. Anal. Appl. 293 (2004), 293-309.

[9] C.L. Frota and N.A. Larkin, Uniform stabilization for a hyperbolic equation with acoustic boundary conditions in simple connected domains, Progr. Nonlinear Differential Equations Appl. 66 (2005), 297-312.

[10] J.Y. Park and J.A. Kim, Some nonlinear wave equations with nonlinear memory source term and acoustic boundary conditions, Numer. Funct. Anal. Optim. 27 (2005), 889-903. 
[11] J.Y. Park and T.G. Ha, Well-posedness and uniform decay rates for the Klein-Gordon equation with damping term and acoustic boundary conditions, J. Math. Phys. 50 (2009), Article No. 013506; doi:10.1063/1.3040185.

[12] A. Vicente, Wave equations with acoustic/memory boundary conditions, Bol. Soc. Parana. Mat. 27 (2009), no. 3, 29-39.

[13] Y.H. Kang, Energy decay rate for the Kirchhoff type wave equation with acoustic boundary condition, East Asian Mathematical Journal 28 (2012), no. 3, 339-345.

[14] Y.H. Kang, Energy decay rates for the Kelvin-Voigt type wave equation with acoustic boundary condition, J. KSIAM. 16 (2012), no. 2, 85-91.

[15] A. Zarai and N.-E. Tatar, Global existence and polynominal decay for a problem Balakrishnan-Taylor damping, Archivum Mathematicum(BRNO). 46 (2010), 157-176.

[16] A.V. Balakishnan and L.W. Taylor, Distributed parameter nonlinear damping models for flight structures, Damping 89, Flight Dynamics Lab and Air Force Wright Aeronautical Labs, WPAFB, 1989.

[17] R.W. Bass and D. Zes, Spillover, nonlinearity and flexible structures, The Fourth NASA Workship on Computational Control of Flexible Aerospace Systems, NASA Conference Publication 10065 (L.W. Taylor, ed.), 1991, 1-14.

Institute of Liberal Education, Catholic University of Daegu, 330 Geumank-Ri, HAyAng-Eup, GyeOngsan-Si GyeOngsan-Si,Gyeongbuk 712-702, Korea

E-mail address: yonghann@cu.ac.kr 